\title{
One-Dimensional Model Study of the PNG Dataset Using Site-Independent Groom-Bailey Decomposition
}

\author{
Hiroaki TOH $^{1}$ and Makoto UYESHIMA ${ }^{2}$ \\ ${ }^{1}$ Ocean Research Institute, University of Tokyo, Tokyo 164, Japan \\ ${ }^{2}$ Earthquake Research Institute, University of Tokyo, Tokyo 113, Japan
}

(Received January 31, 1995; Revised December 5, 1995; Accepted June 3, 1996)

\begin{abstract}
Conventional magnetotelluric (MT) analyses of the Papua New Guinea (PNG) dataset reveal that it is a very good two-dimensional (2D) dataset with relatively high $\mathrm{S} / \mathrm{N}$ ratios up to $10^{2} \mathrm{~s}$. However, traditional Swift's angles are unstable at some sites and differ slightly site to site. This implies that weak but significant distortions are present at all sites. Hence, the regional $2 \mathrm{D}$ strike angle was sought by minimizing the global $\chi^{2}$ misfits derived from the site- and frequency-independent Groom-Bailey (GB) decomposition at seven selected sites. The other three sites were eliminated by $\chi^{2}$-test. The strike angle was determined to be $60^{\circ}$ west of geographic north. One-dimensional (1D) Monte-Carlo inversion was performed using the Berdichevsky averages of the decomposed 2D principal tensor elements. The 2D model simply based on the 1D inversions was able to reproduce the observed impedances, except for the systematically high values of TM phase in the period range of $10^{-1}$ to $10 \mathrm{~s}$. This indicates that a 1D approximation of the electrical conductivity structure beneath this region is reasonably valid. The 1D models indicated the existence of a thin (up to $2.5 \mathrm{~km}$ ) resistive layer just below the surface, suggesting the 'single' Darai limestone.
\end{abstract}

\section{Introduction}

In this paper, we present 1D inversions of the PNG dataset. The validity of the 1D models derived is examined by 2D forward modeling. To achieve this, it is essential to derive 'good' 1D or regional 2D responses from the magnetotelluric (MT) observations. Removal of threedimensional (3D) surface distortions, based on simple physical models has been widely discussed in recent literature by Bahr (1988, 1991), Groom and Bailey $(1989,1991)$, Groom and Bahr (1992), Groom et al. (1993) and references therein. Using the Groom and Bailey $(1989,1991)$ (GB) method, MT data collected at a specific site can be modeled either by frequency-dependent, or by frequency-independent, decomposition even in the presence of strong 3D distortions, as in case of the BC87 dataset (Jones et al., 1993). However, site-independent GB decomposition for a whole dataset has never been attempted although it is very important in constructing a self-consistent $2 \mathrm{D}$ model.

\section{Data}

The PNG dataset comprises 10 sites of MT data, collected in the southeastern part of PNG. The traverse line was approximately $5.6 \mathrm{~km}$ in length, oriented in a SSW-NNE direction. Observations were made at up to 40 frequencies, ranging from $384 \mathrm{~Hz}$ to $1820 \mathrm{~s}$, although large error bars at low frequencies indicated that the impedance estimates at these frequencies were not reliable. Hence, MT responses to a maximum period of $909.9 \mathrm{~s}$ were used, and only 38 frequencies were considered in this study. Tipper values were not used in our analysis as they showed large scatter and little consistency with MT impedances, which may be due to problems with the $B z$ measurements. A traditional measure of three-dimensionality, known as skewness, 


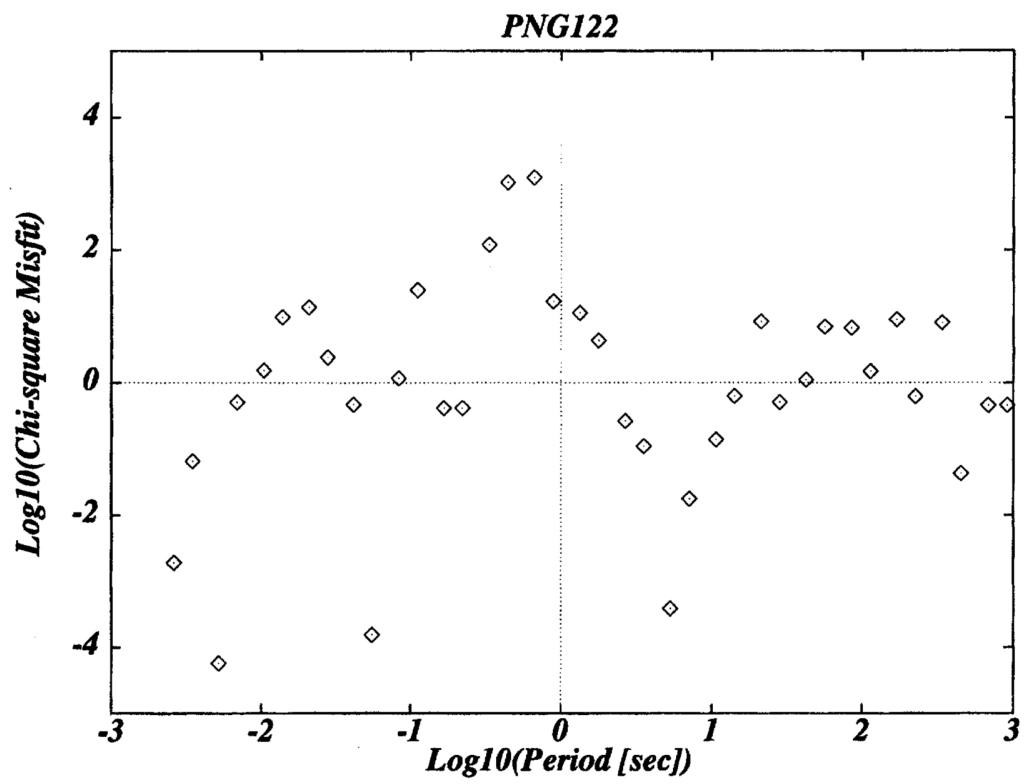

(a)

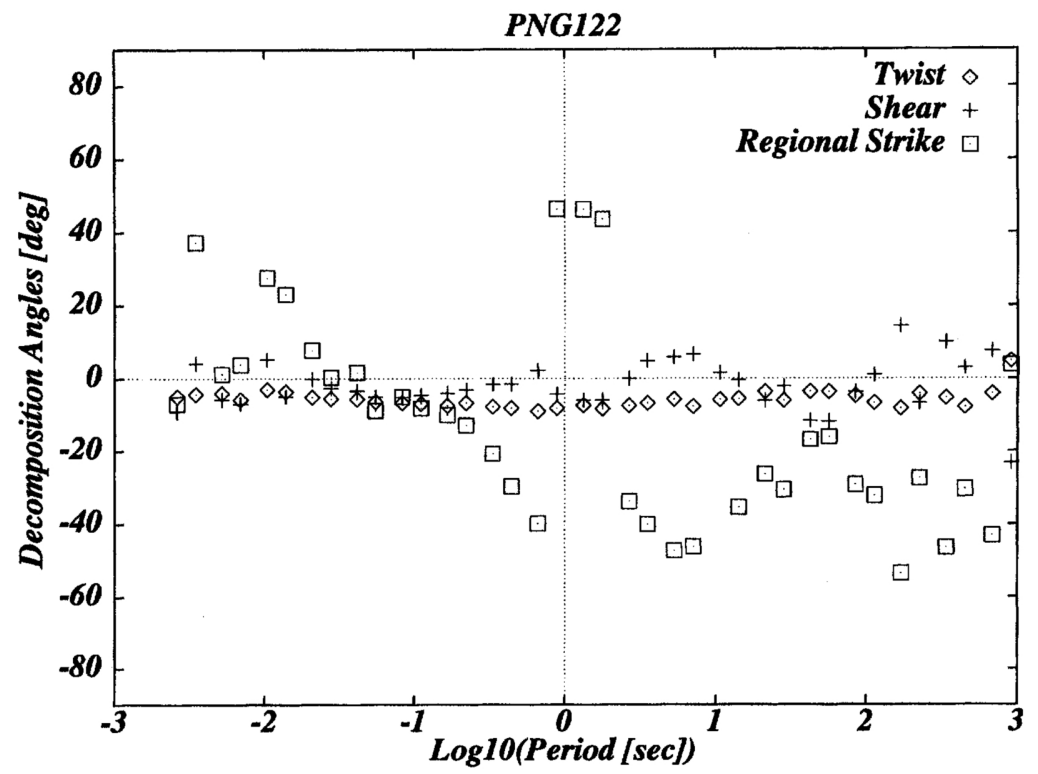

(b)

Fig. 1. Results of the frequency-dependent GB decomposition at site PNG122. (a) $\chi^{2}$ misfits. (b) Twist, shear and $2 \mathrm{D}$ regional strike angles. (c) Observed $Z x x$ and $Z x y$ impedances. Solid lines are those predicted by the GB decomposition. (d) Same diagram as (c) for $Z y x$ and $Z y y$. 

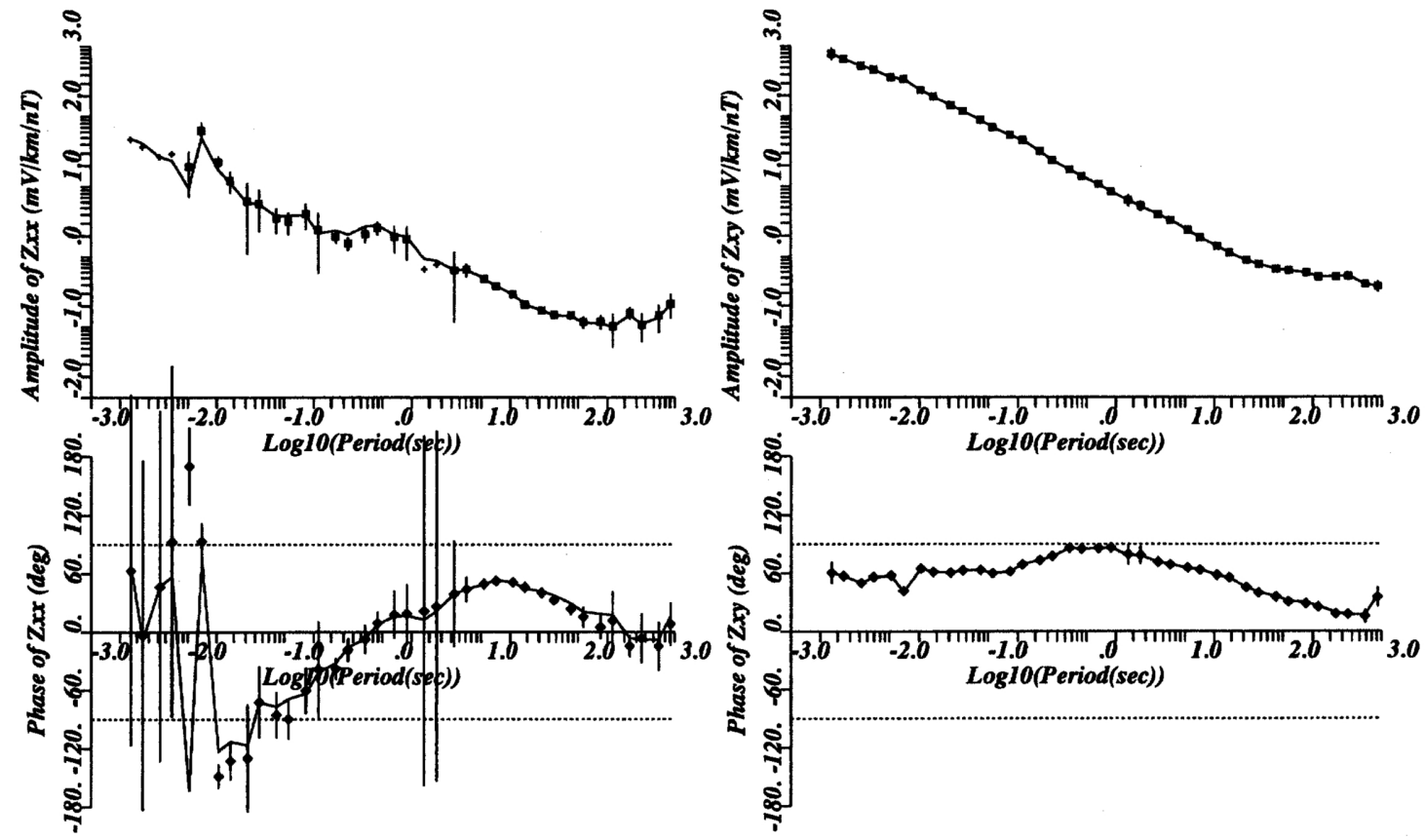

(c)
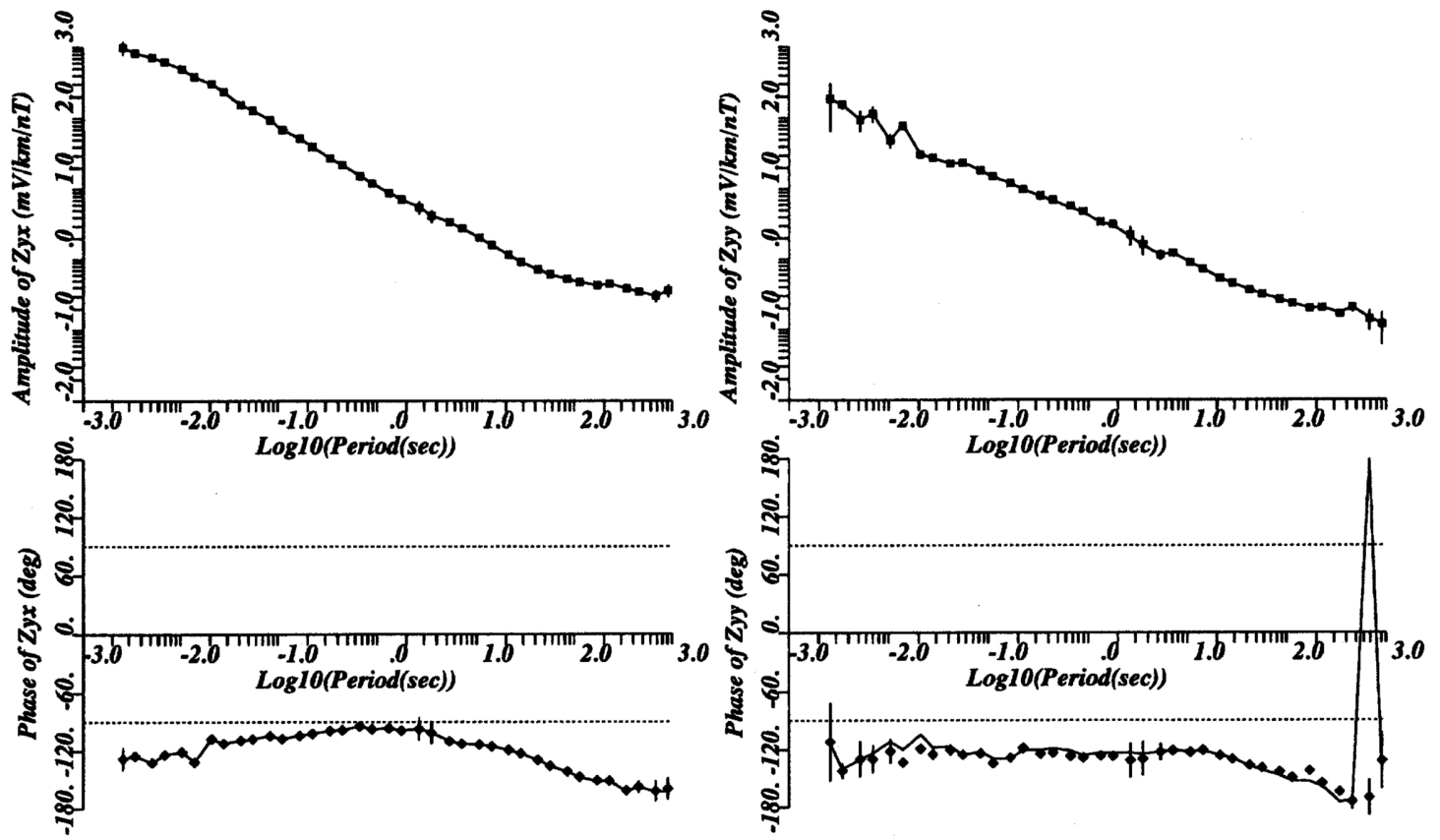

(d)

Fig. 1. (continued). 
gives small skew values of less than 0.2, except at the lowest frequencies and at site PNG121. Additionally, the diagonal elements of the impedance tensor $Z x x$ and $Z y y$ are very small in comparison with the off-diagonal elements $Z x y$ and $Z y x$. This evidence suggests that the PNG dataset is approximately ' $2 \mathrm{D}$ ', and is therefore suitable for constructing a detailed regional $2 \mathrm{D}$ electrical conductivity structure beneath the region concerned.

The regional 2D strike angle of the PNG dataset was found to coincide approximately with the direction perpendicular to the profile (SSW-NNE). For example, using Swift's method to calculate strike angles, at site PNG101 a frequency-independent strike of $60^{\circ}$ west of geographic north was found. However, as is often the case with MT data, the regional strike angle is a variable parameter, differing site by site and with frequency (cf. Jones and Groom, 1993). In fact, Swift's angles at site PNG104 are very variable in this frequency range. We, therefore, judged that the MT impedance tensors of the PNG dataset were subject to weak but significant near-surface 3D distortion and decided to apply GB decomposition to the PNG dataset.

Another point that should be noted here is a TM mode phase anomaly between $10^{-1}$ to 10 $\mathrm{Hz}$, where phases are very high and sometimes larger than $90^{\circ}$. It is very difficult to interpret this anomaly by 1D and/or 2D models other than by introducing the concept of anisotropy into the conductivity structure, as had been presented by M. Eisel at MT-DIW2 in Cambridge. This problem will be further discussed later in this paper.

\section{Decomposition}

Tensor GB decomposition of the PNG dataset was carried out with the following three steps using the GB method. First, frequency-dependent GB decomposition at each site was performed. Then, we proceeded to frequency-independent GB decomposition at each site, from which ten 2D regional strike angle estimates were obtained. Finally, one site-independent $2 \mathrm{D}$ regional strike angle was determined by using the data at seven sites selected by $\chi^{2}$-test. Only electric field distortion was considered in any of our GB decompositions.

\subsection{Frequency-dependent GB decomposition}

Frequency-dependent GB decomposition, which allows the decomposition parameters such as twist, shear and regional strike angles to vary in the frequency domain, gave smaller $\chi^{2}$ misfits at each site (Fig. 1(a)) than frequency-independent GB decomposition. The 2D regional strike angles determined at each site are not consistent with other sites and are frequency-dependent. Strike angles are often very variable even when the twist and shear angles are relatively invariant with frequency (Fig. 1(b)). The modeled fit to the observed data (Figs. 1(c) and 1(d)) clearly shows that predicted values from frequency-dependent GB decomposition are likely to follow every data point including outliers. It is worth noting that it would be useful to build in some element of robustness into the decomposition, though elimination of simple isolated outliers may be more easily accomplished in the course of response function estimation using robust codes.

\subsection{Frequency-independent $G B$ decomposition}

Next, decomposition parameters were fixed in the frequency range concerned. This gave larger $\chi^{2}$ misfits and the 2D strike angle estimates were still local in the sense that they differed site-by-site. Figures $2(\mathrm{a})$ and $2(\mathrm{~b})$ show the site-dependency of the $2 \mathrm{D}$ regional strike angle estimates at all sites and their $\chi^{2}$ misfits, respectively. It is evident from the figures that strike angles at sites PNG102, 121 and 122 are much different from other sites. In addition, the $\chi^{2}$ misfits of the three sites are very large in comparison with those of the other sites. 


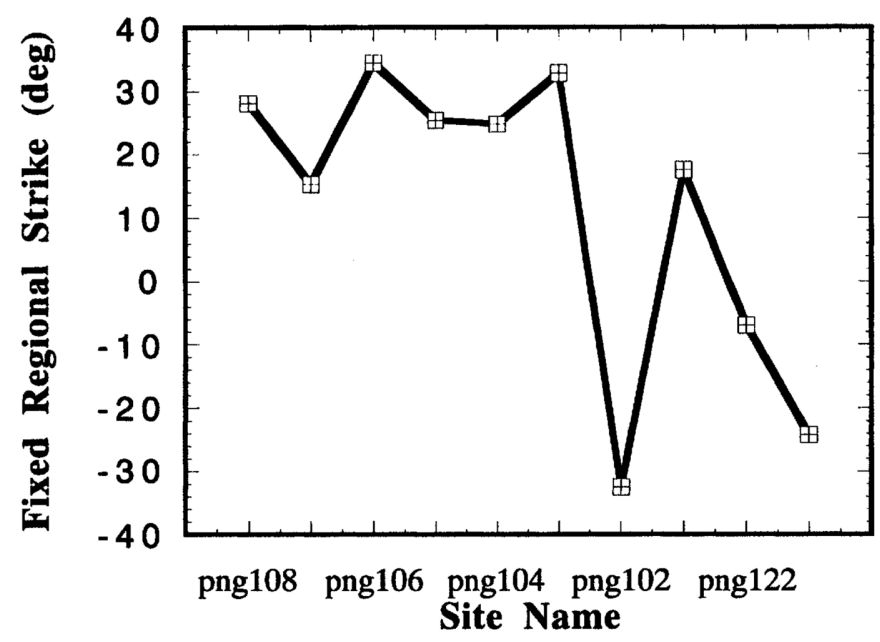

(a)

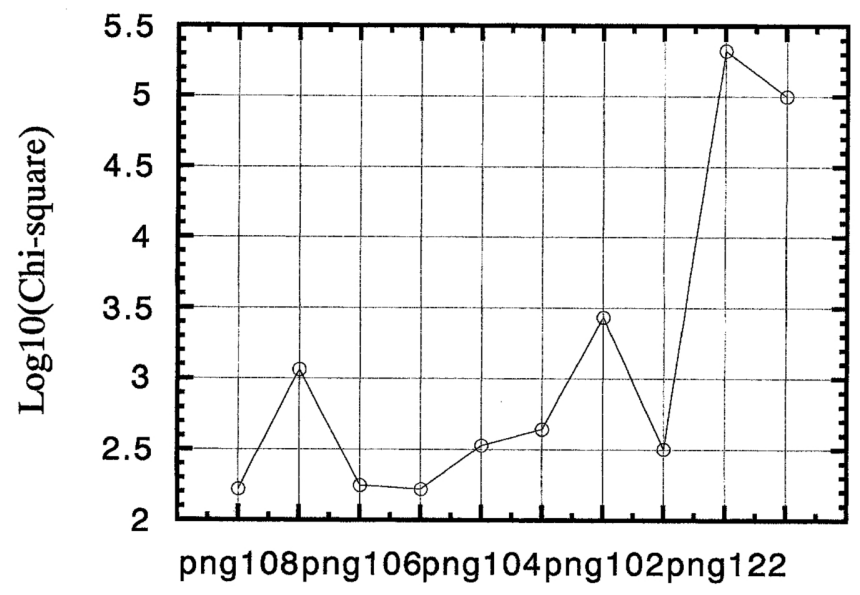

(b)

Fig. 2. Results of the frequency-independent GB decompositions. (a) The 2D regional strike angle estimates of all the sites. (b) Respective $\chi^{2}$ misfits.

\subsection{Site-independent $G B$ decomposition}

The final procedure of determining both site- and frequency- independent $2 \mathrm{D}$ strike was performed as follows. (1) Apply frequency-independent GB decomposition for a given regional strike angle to all the sites. (2) Calculate the sum of the $\chi^{2}$ misfits. (3) The given regional strike angle in (1) was changed from $0^{\circ}$ to $90^{\circ}$ by $1^{\circ}$ interval, and the best angle was determined so that the sum of $\chi^{2}$ misfits in (2) took the minimum value.

First, we applied steps (1) to (3) to all the sites. The best angle was determined to be $72^{\circ}$ with the $\chi^{2}$ misfits of 188,689 , which was much larger than its $99 \%$ confidence limit of 3224.36 . Then, we successively eliminated bad sites with large $\chi^{2}$ misfits and performed the process (1) (3) again. The sum of $\chi^{2}$ misfits did not decrease below its $99 \%$ confidence limit until the $\chi^{2}$ misfits 


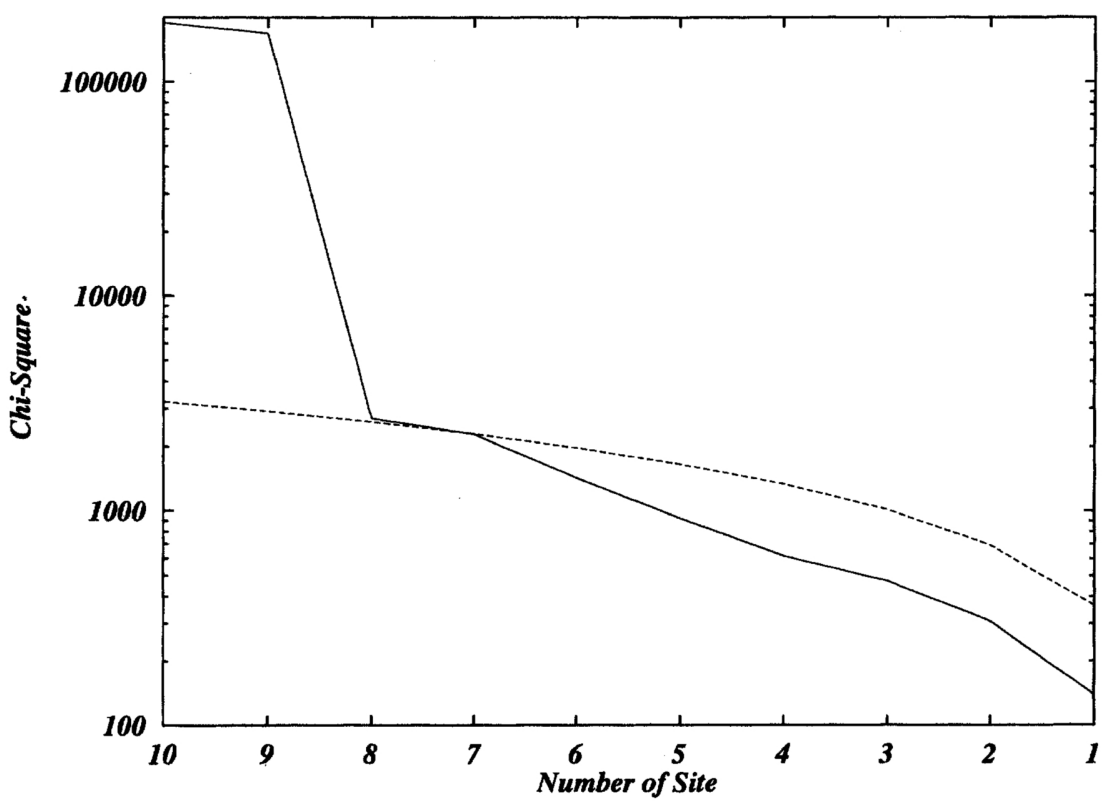

Fig. 3. Statistical test of the $\chi^{2}$ misfits. The solid line is the sum of the $\chi^{2}$ misfits while the dashed line shows the $99 \%$ confidence limit. $X$-axis is number of sites used in the summation. In this test, sites with larger misfits were removed from the summation one by one. The sum of the $\chi^{2}$ misfits did not become smaller than respective $99 \%$ confidence limits until sites PNG102, 121 and 122 were removed.

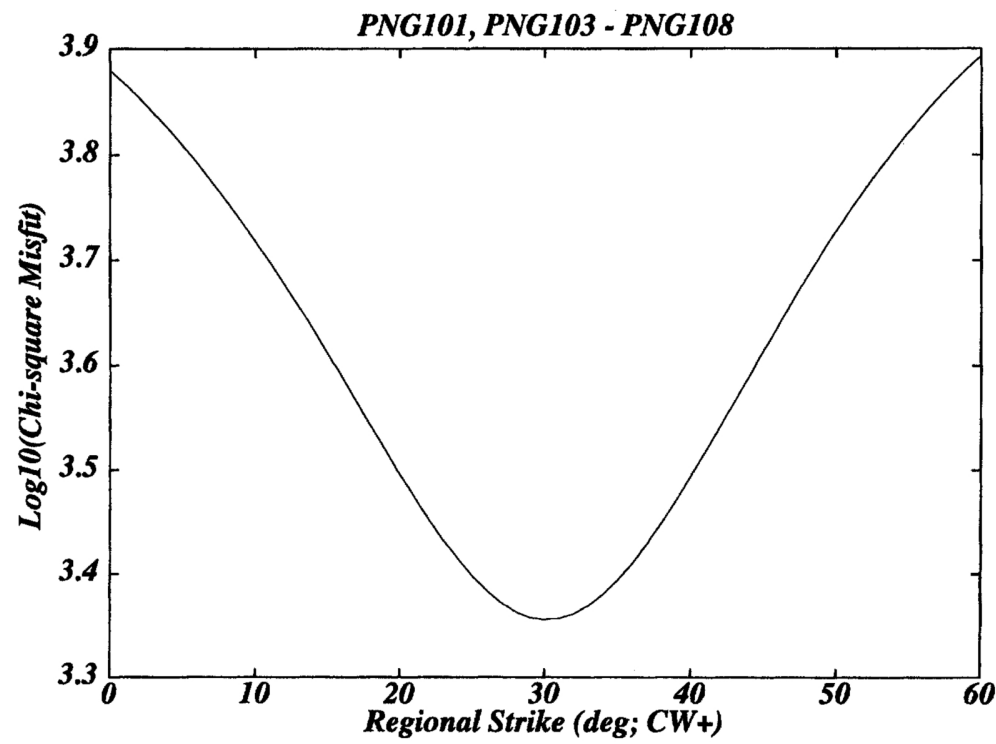

Fig. 4. Plot of 2D regional strike angles vs. global $\chi^{2}$ misfits derived by the site-independent GB decomposition. The strike angles were measured clockwise from the geographic north. 

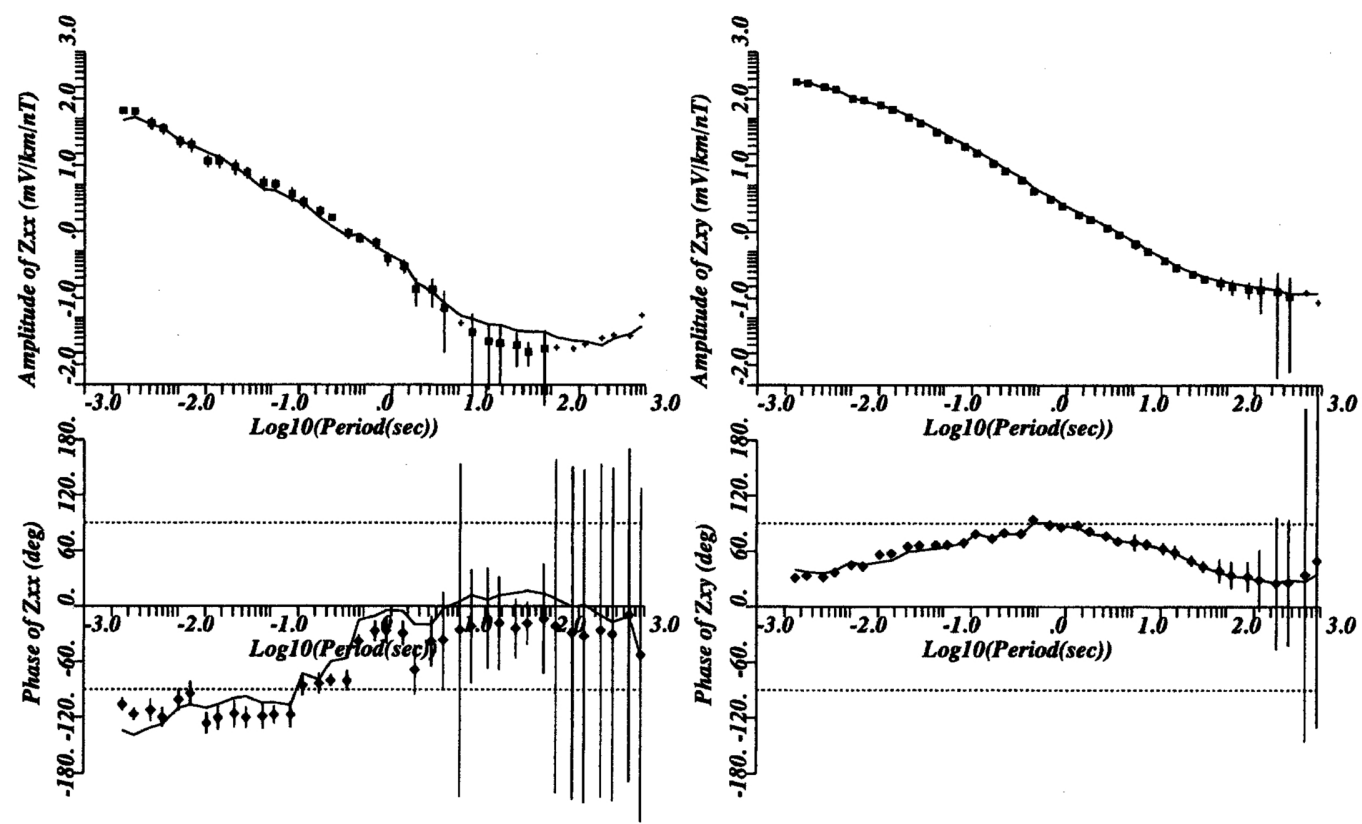

(a)
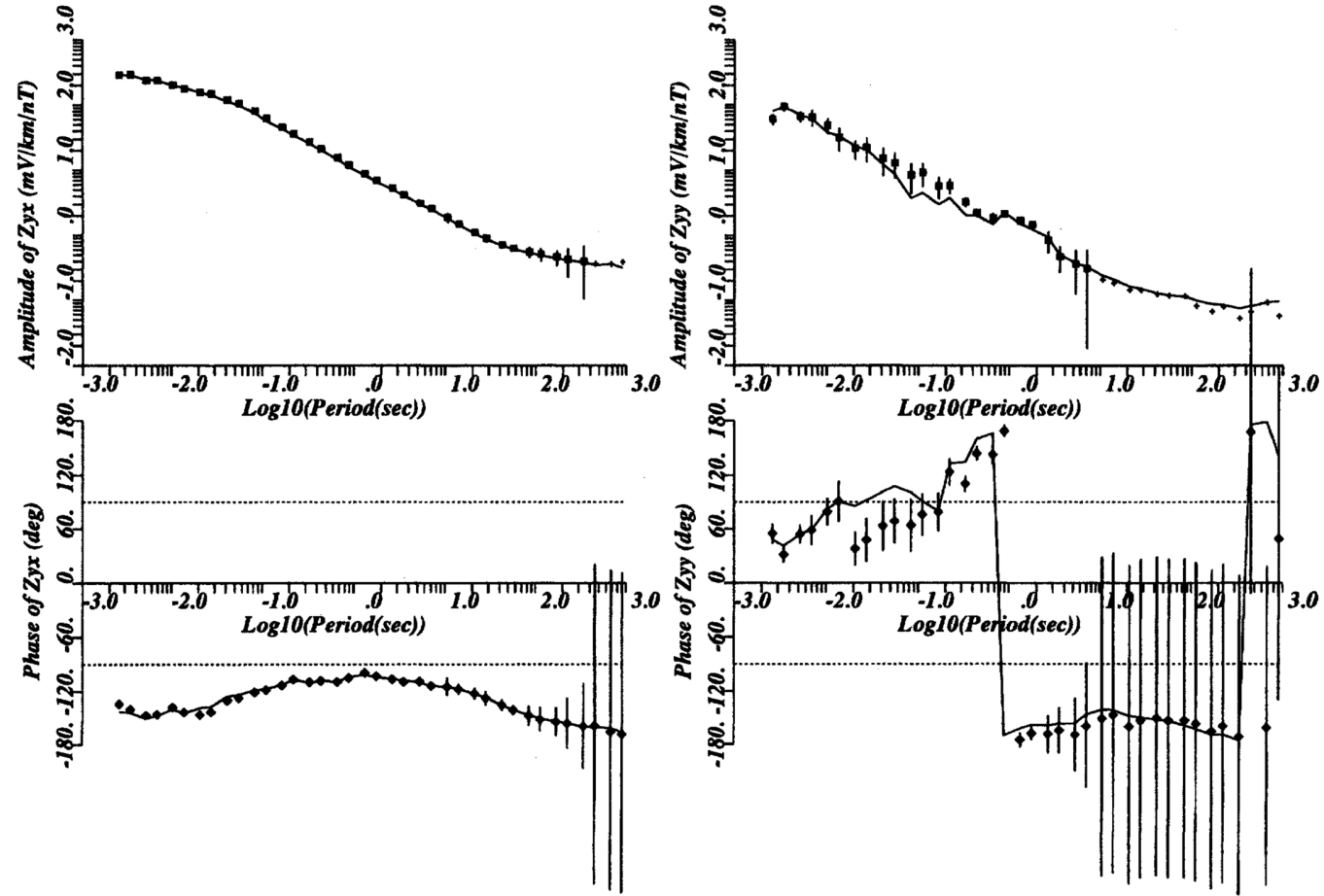

(b)

Fig. 5. Results of the site-independent GB decompositions at site PNG102. (a) Observed $Z x x$ and $Z x y$ impedances (symbols with error bars). Solid lines are those predicted by the GB decomposition. (b) Same diagram as (a) for $Z y x$ and Zyy. 
for the sites PNG102, 121 and 122 were removed from the summation (Fig. 3), which suggested that $\chi^{2}$ misfits of these three sites were statistically insignificant. Accordingly, these three sites were discarded from the determination of the true $2 \mathrm{D}$ regional strike angle. The angle was finally determined to be $60^{\circ}$ west of geographic north (Fig. 4). The global $\chi^{2}$ misfit was 2269.18 which was smaller than its confidence limit of 2282.73. We regarded this angle as the $2 \mathrm{D}$ regional strike of the PNG dataset and used it in our subsequent analyses. Model fit to the observed data using this regional strike angle was shown in Figs. 5(a) and 5(b).

\section{1D Inversion}

1D inversions were conducted using the arithmetic means (Berdichevsky and Dmitriev, 1976) of the off-diagonal elements of the impedance tensor derived by the site-independent GB decomposition. We used H. Utada's MT 1D inversion code (Utada, 1987) which was based on a Monte Carlo search for minimum variance. We considered the possibility that the PNG dataset might be affected by source-field effects, as it was collected near the equatorial region. However, the verification of the $P_{1}^{0}$ approximation was first conducted by changing the source-field wave-lengths. It was found that longer wave-lengths gave better fits to the observed $1 \mathrm{D}$ responses, implying the absence of significant source-field effects in the PNG dataset.

It was essential in our 1D inversions to determine the number of layers required at each site. A preliminary run of the 1D code, using 100 Monte Carlo trials at each site, was carried out, varying the number of layers from 2 to 10 . Figure 6 shows a plot of variance with number of layers. It is clear from the figure that the calculated variances converged using either 4 layer or 5 layer model, and adding more layers did not significantly reduce the variances.

$1 \mathrm{D}$ electrical conductivity models of all the 10 sites were then refined by subsequent re-runs of the $1 \mathrm{D}$ code using 2000 Monte Calro trials at each site, with either a 4 or 5 layer model. The results of the inversions are shown in Fig. 7. Note that the phase anomaly is particularly evident at sites PNG102, 121 and 122, but other sites, such as PNG101 and 103, are not necessarily free

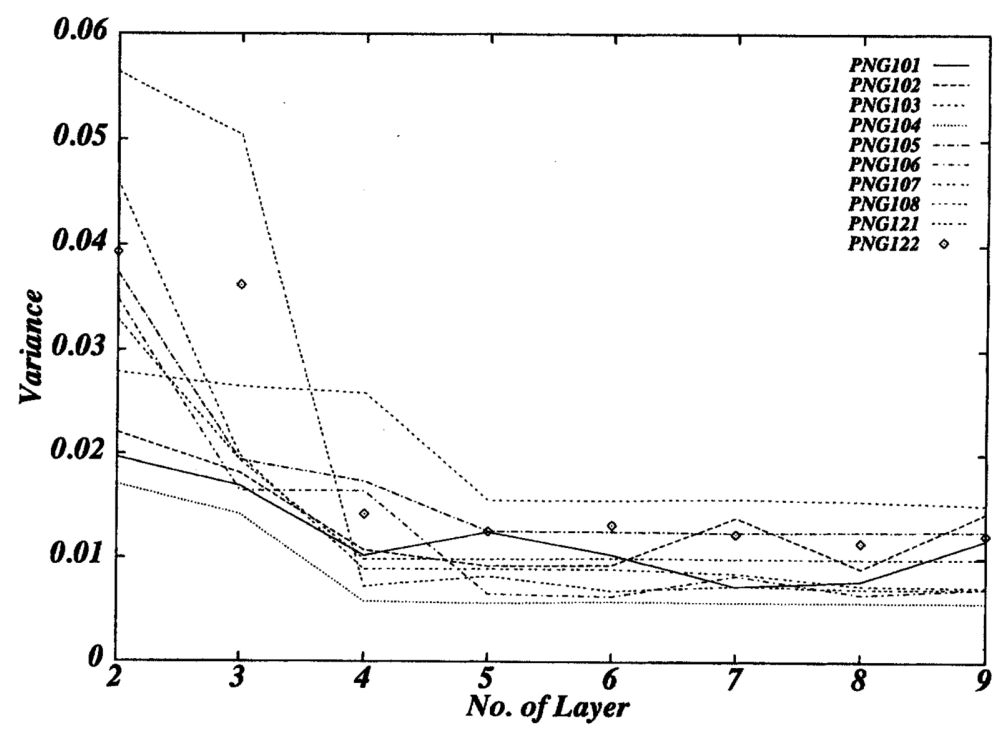

Fig. 6. Variance vs. the number of layers of the 1D models. Convergence to significantly low levels has reached at either 4- or 5-layered models. 

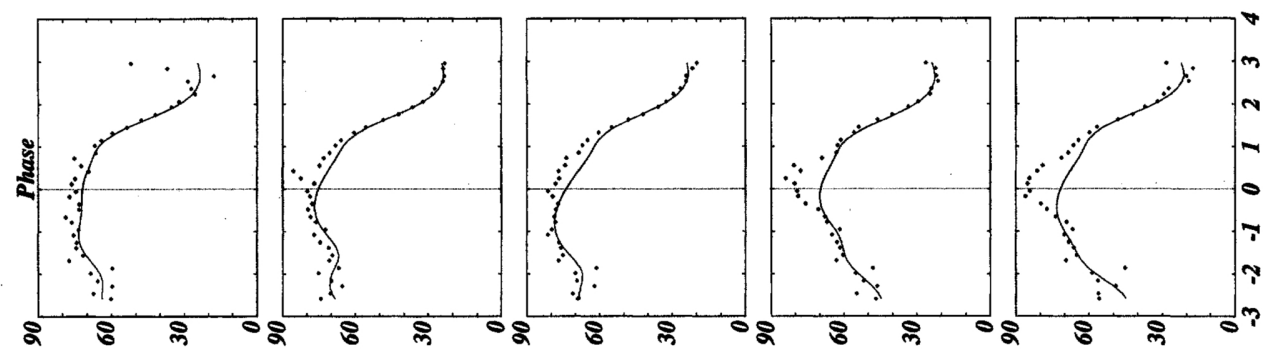

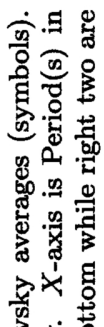
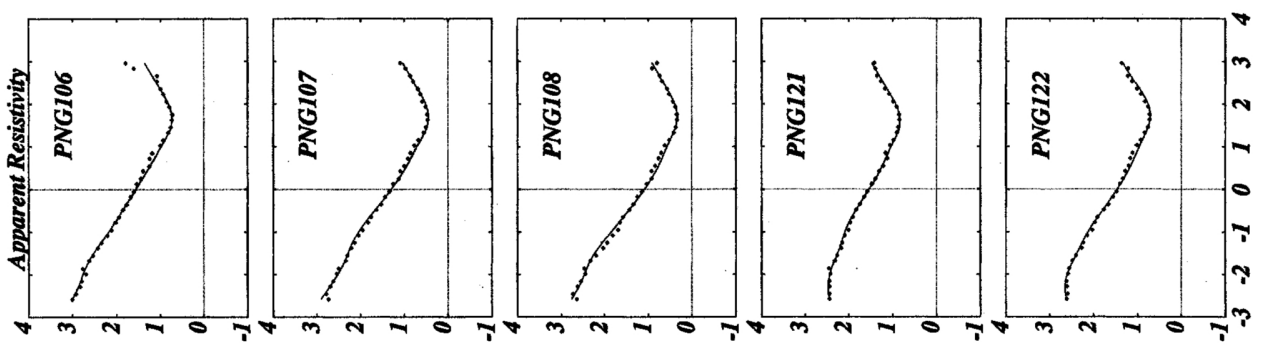

突苛

氕?

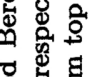

密 की

옹명

ठ용

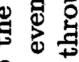

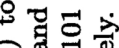

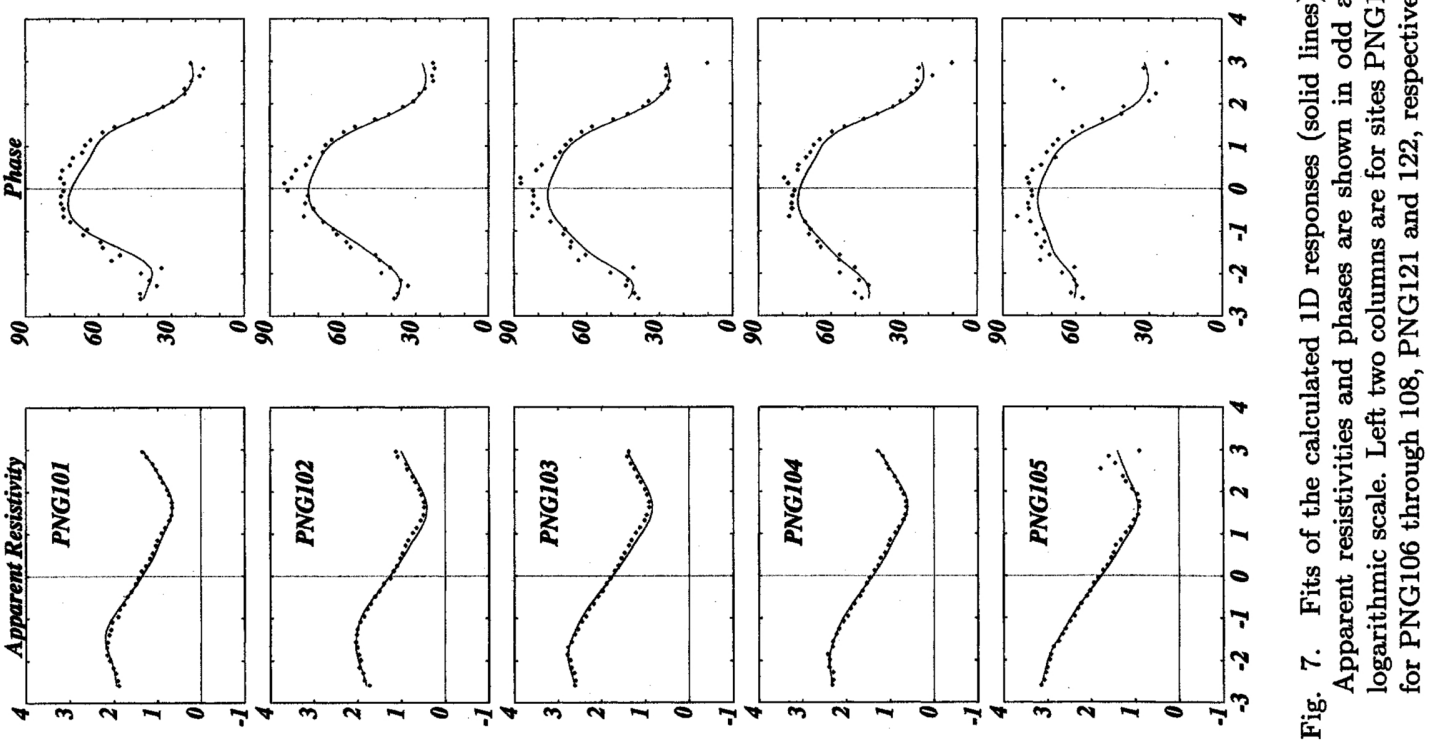




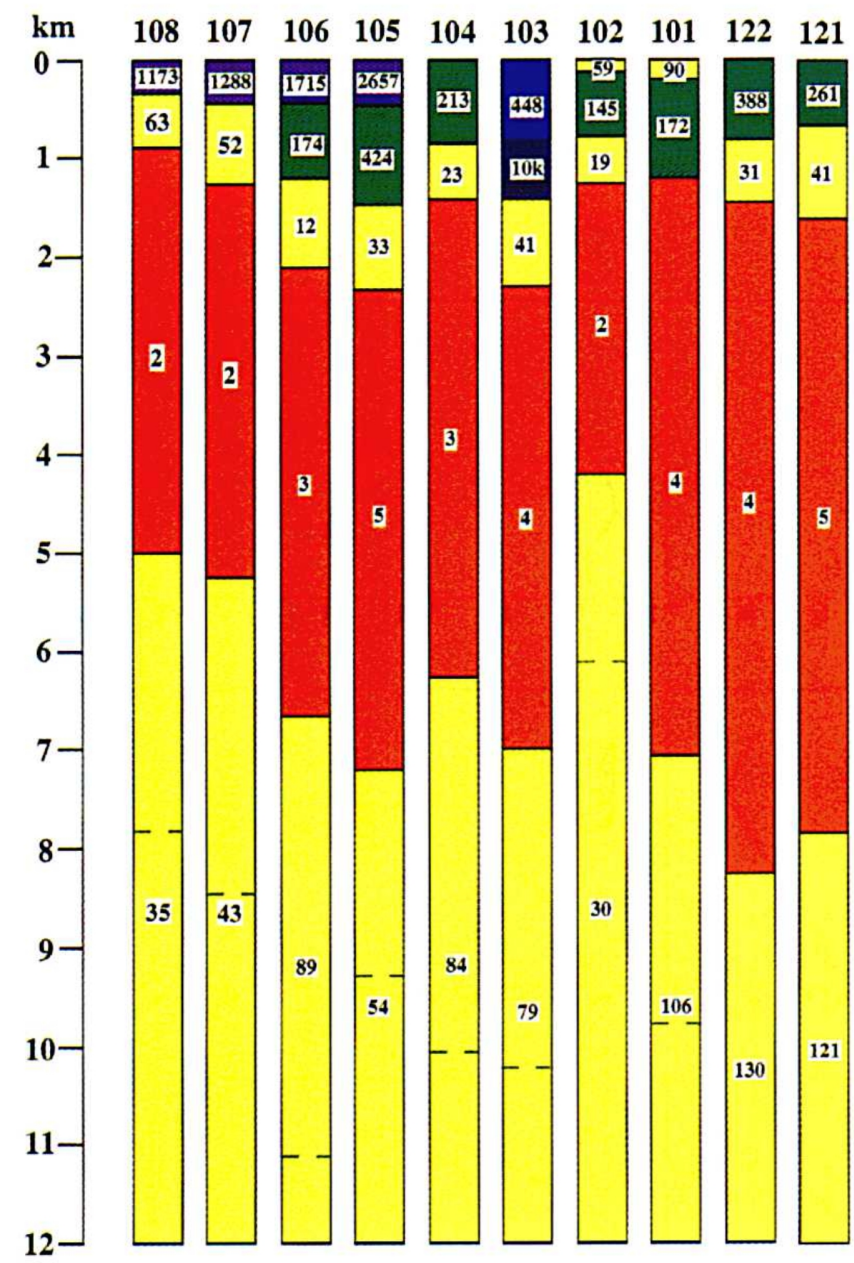

Fig. 8. Ten columnar 1D models derived by the Monte Carlo inversion. $Y$-axis is depth (km) in linear scale. The columns are arranged from the southernmost site (PNG108) to the northernmost site (PNG121). Numerals in each column are resistivities in ohm $\cdot \mathrm{m}$.

from the phase anomaly. Figure 8 shows the $1 \mathrm{D}$ models of the 10 sites.

In our final 1D models, a single resistive layer was found beneath the surface. The resistive layer dips to NNE (though it is not clear at sites PNG102 and 104) and gradually reduces its resistivity at depths. The thickness of the resistive layer is at most $2.5 \mathrm{~km}$. Very conductive sediments, which may be a source of hydrocarbons, of 3 to $6 \mathrm{~km}$ thickness and resistivities of 2 to $5 \mathrm{ohm} \cdot \mathrm{m}$ was found beneath the resistive layer. These features do not coincide with the 'double Darai limestone' based on the geological constraints, provided that the surface resistive portion is interpreted as the Darai limestone.

\section{Discussion}

In the course of our site-independent GB decomposition, sites PNG102, 121 and 122 were detected by a $\chi^{2}$-test as containing significant outliers. As shown in Figs. 5 (a) and 5(b), the observed values at site PNG102, especially the phases of diagonal elements $Z x x$ and $Z y y$, contain 

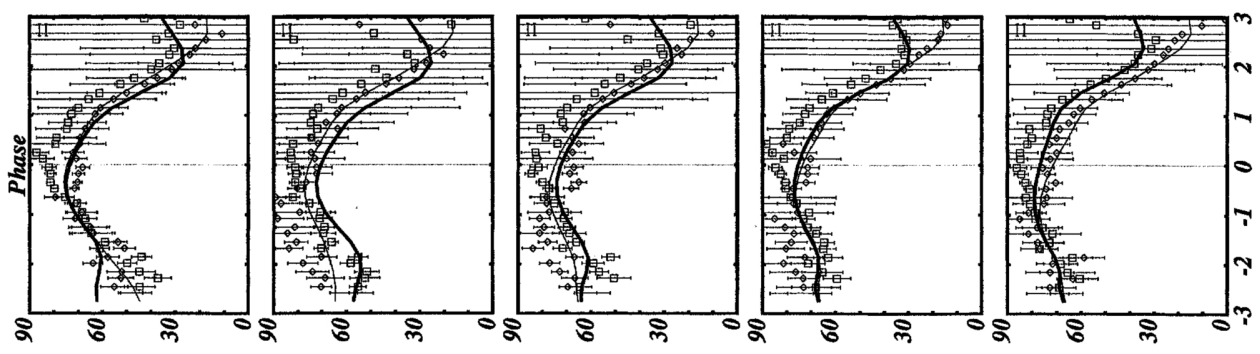

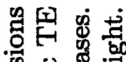

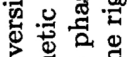

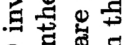

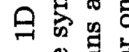

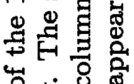

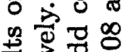

武获

㠃

क्ष

ธี ซึ.

范苛罢

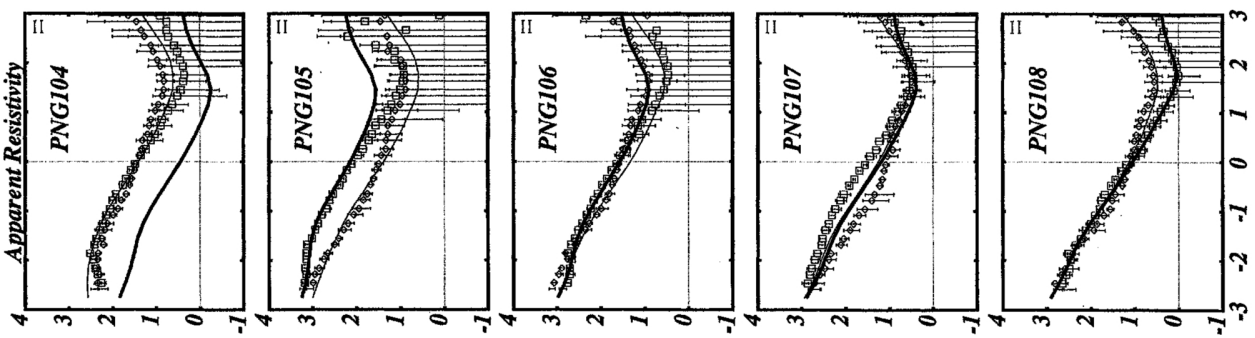

형됴웡

品范

ล

肎总范

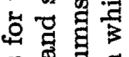

원

ठํㅕㅇㅇㅛ

을 国
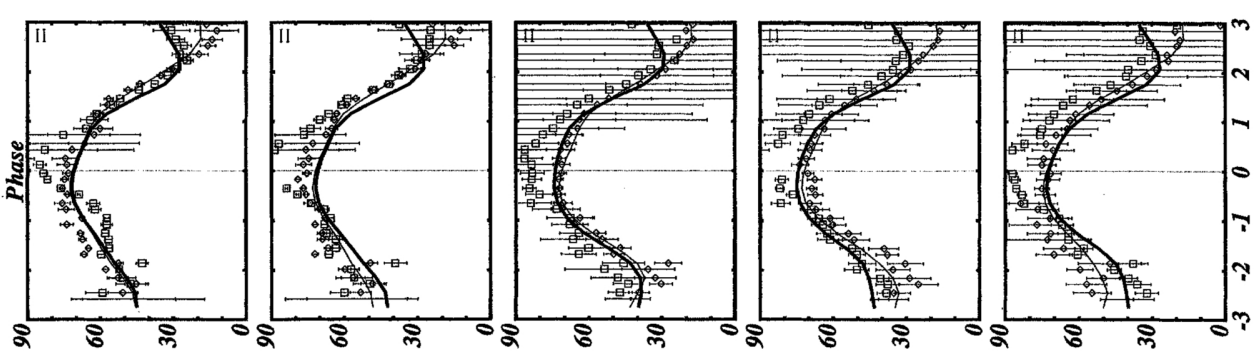

它家

월

兒总总

.

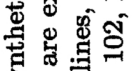

형

ซี ซิำ สิ

娄

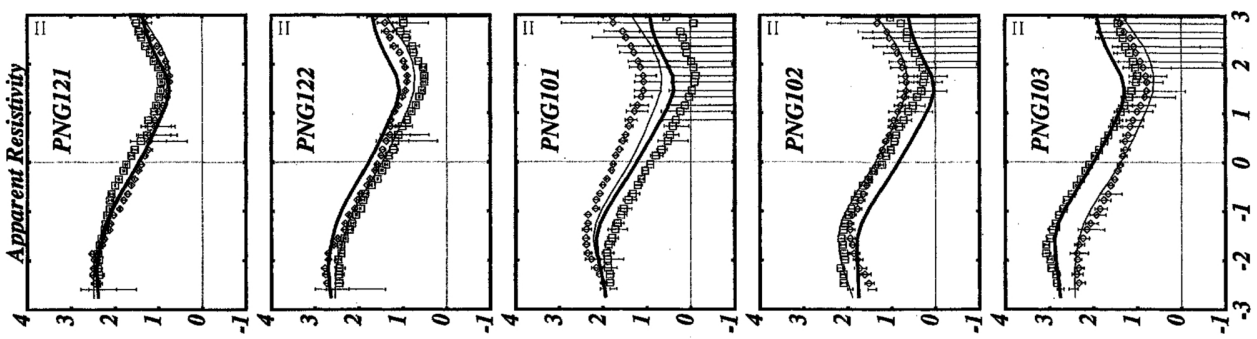

卷击望

\&

氙牙总递

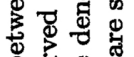

员芯

.

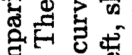

ठิ宀

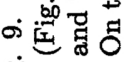
뭉 

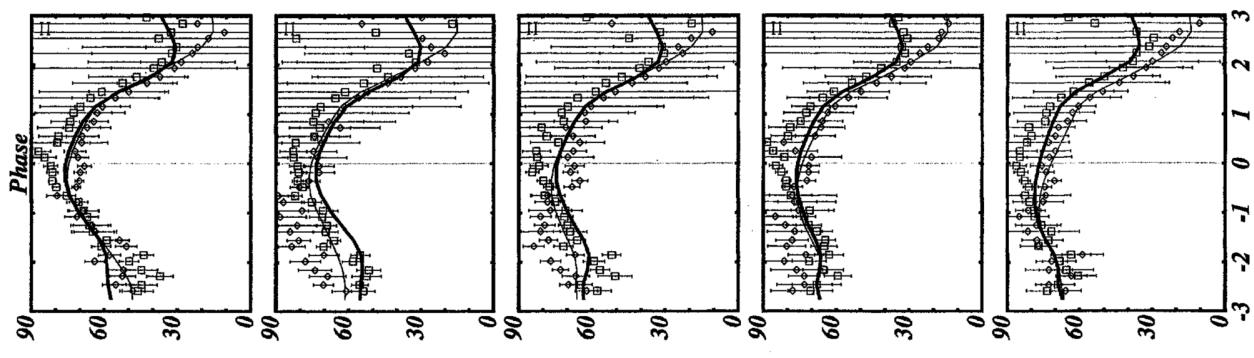

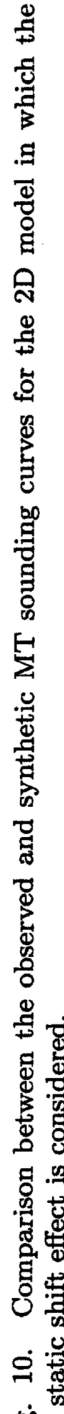
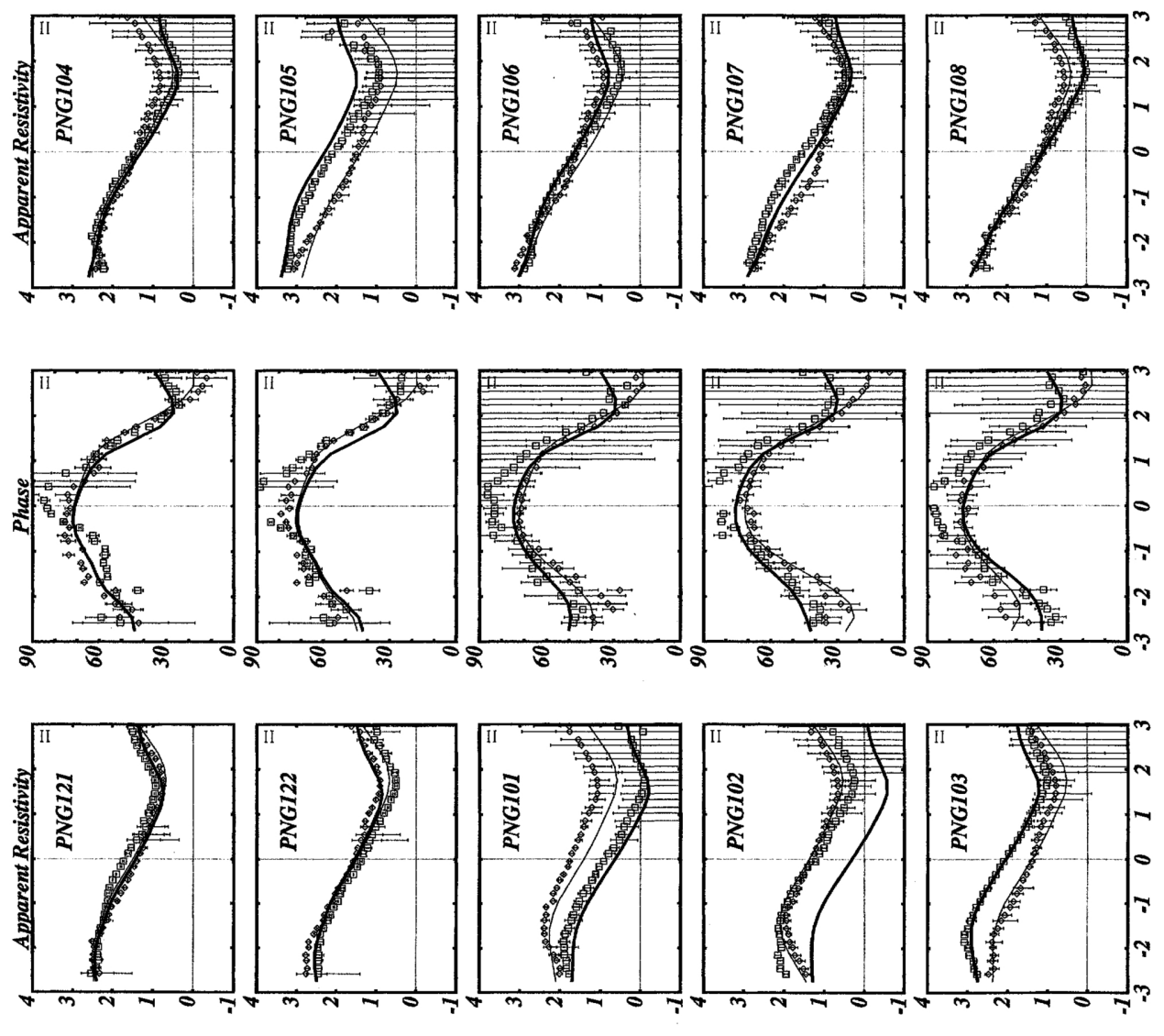
large scatter and steps which cannot be explained by their error estimates. True MT responses must vary smoothly in the frequency domain. Thus, the scatter and steps may possibly be due to contamination of significant coherent noise. On the other hand, the significant discrepancies between the synthetic and observed impedances often coincides with the scatter and steps. Those discrepancies cannot be explained by their error estimates, either, which may result in large $\chi^{2}$ misfits. Similar tendencies can be seen at sites PNG121 and 122 as well. Accordingly, the validity of the elimination of the three sites by $\chi^{2}$-test is confirmed.

We only minimized 'data misfit' in search for the true 2D strike angle estimate. However, this method can be extended to some regulated minimizations including 'model roughness'. For example, ABIC minimization (Uchida, 1993) can handle, in a more statistical manner, the $a$ priori information that the true 2D regional strike angle, if any, should be site- and frequencyindependent, or, in a more practical sense, differ very slightly site by site and in the frequency domain. Such regulated minimizations is likely to give more reliable estimates of the true $2 \mathrm{D}$ strike angle.

As for our 1D model, there are three problems. First, there is no theoretical reason why the Berdichevsky average can be used for the 1D modeling. Second, 1D inversion without static shift correction is unrealistic. Third, the systematic discrepancy between the synthetic phases and the observed values remained in the period range of $10^{-1}$ to $10 \mathrm{~s}$ (Fig. 7). In order to examine these problems, we constructed a 2D model simply based on stitching together the 1D inversions (Fig. 8). A 2D finite-element method (Utada, 1987) was used in this study. In Fig. 9, synthetic apparent resistivities and phases are compared with the observations. Although this model was only based on the best fit $1 \mathrm{D}$ models, the observations are generally well reproduced, especially for the TE mode. As for the TM mode apparent resistivities, a delicate adjustment to the observations without any change in phases was achieved by incorporating static shifts. The result is shown in Fig. 10, which was obtained by changing the near surface resistivity values and width of the 1D columns. Note that the apparent resistivities at PNG101 and 104 are much improved in comparison with those in Fig. 9 without changing the major features of the $1 \mathrm{D}$ models shown in Fig. 8. Thus, our 1D models are proved to be representations of the vertical structure below the respective site. However, the last problem still remais. The $2 \mathrm{D}$ modeling also showed that the main discrepancy lay in the systematically high values of the observed TM phases, viz., the TM phase anomaly could not be reproduced even in our $2 \mathrm{D}$ models. Introducing the anisotropic resistivity structure is one possible way to solve this problem.

\section{Conclusions}

The 2D regional strike angle of the PNG dataset was determined as $60^{\circ}$ west of geographic north by site- and frequency-independent GB decomposition using data from the seven selected sites. The other three sites were excluded by $\chi^{2}$ test. It was also easily understood by the visual inspection that the data at the three sites contained many outliers. The present method may be helpful for determining site- and frequency-independent regional strike and the examination of its existence.

1D inversions were conducted at each site using the Berdichevsky averages of the 2D principal tensor elements derived by the site-independent GB decomposition. The validity of the 1D models was tested by the 2D forward modeling based on the 1D models. As a result, our 2D model was also able to reproduce the observed impedances, except for the systematic upheaval of TM phases.

In our final 1D model, a thin $(\sim 2.5 \mathrm{~km})$ resistive layer varying slightly in thickness was detected just below the surface. A very conductive layer of 3 to $6 \mathrm{~km}$ thickness was found underneath. This is not compatible with the 'double' Darai limestone. Hence, it can be concluded that the Darai limestone is 'single'. 
We are grateful to Drs. Charles Swift and Alan G. Jones for providing us with the PNG dataset. We also appreciate Dr. Adam Schultz for his kind help at MT-DIW2 in Cambridge. We also express our sincere thanks to two reviewers, Drs. J. Segawa, Y. Sasai and G. Heinson, and to the external reviewers for their critical review and their valuable suggestions for improving the manuscript. The GB decomposition code was made available to us via 'mtnet.europe'.

\section{REFERENCES}

Bahr, K., Interpretation of the magnetotelluric impedance tensor: Regional induction and local telluric distortion, J. Geophys., 62, 119-127, 1988.

Bahr, K., Geological noise in magnetotelluric data: A classification of distortion types, Phys. Earth Planet. Inter., 66, 24-38, 1991.

Berdichevsky, M. N. and V. I. Dmitriev, Basic principles of interpretation of magnetotelluric sounding curves, in Geoelectric and Geothermal Studies, edited by A. Adam, pp. 165-221, KAPG Geophys. Monogr., Akademiai Kiado, 1976.

Groom, R. W. and R. C. Bailey, Decomposition of magnetotelluric impedance tensors in the presence of local three-dimensional galvanic distortion, J. Geophys. Res., 94, 1913-1925, 1989.

Groom, R. W. and R. C. Bailey, Analytic investigations of the effects of near-surface three-dimensional galvanic scatterers on MT tensor decompositions, Geophysics, 56, 496-518, 1991.

Groom, R. W. and K. Bahr, Corrections for near surface effects: Decomposition of the magnetotelluric impedance tensor and scaling corrections for regionalresistivities: A tutorial, Surv. Geophys., 13, 341-379, 1992.

Groom, R. W., R. D. Kurtz, A. G. Jones, and D. E. Boerner, A quantitative methodology to extract regional magnetotelluric impedances and determine the dimension of the conductivity structure, Geophys. J. Int., 115, 1095-1118, 1993.

Jones, A. G. and R. W. Groom, Strike angle determination from the magnetotelluric tensor in the presence of noise and local distortion: Rotate at your peril !, Geophys. J. Int., 113, 524-534, 1993.

Jones, A. G., R. W. Groom, and R. D. Kurtz, Decomposition and modeling of the BC87 dataset, J. Geomag. Geoelectr., 45, 1127-1150, 1993.

Uchida, T., Smooth 2-D inversion for magnetotelluric data bsed on statistical criterion ABIC, J. Geomag. Geoelectr., 45, 841-858, 1993.

Utada, H., A direct inversion method for two-dimensional modeling in the geomagnetic induction problem, $\mathrm{Ph} . \mathrm{D}$. Thesis, Univ. Tokyo, 409pp., 1987. 